This item was submitted to Loughborough's Research Repository by the author.

Items in Figshare are protected by copyright, with all rights reserved, unless otherwise indicated.

\title{
Using buffers in trust aware relay selection networks with spatially random relays
}

PLEASE CITE THE PUBLISHED VERSION

http://doi.org/10.1109/TWC.2018.2850016

PUBLISHER

(C) IEEE

VERSION

AM (Accepted Manuscript)

LICENCE

CC BY-NC-ND 4.0

\section{REPOSITORY RECORD}

Gong, Yu, Gaojie Chen, and Tian Xie. 2018. "Using Buffers in Trust Aware Relay Selection Networks with Spatially Random Relays”. Loughborough University. https://hdl.handle.net/2134/33731. 


\title{
Using Buffers in Trust Aware Relay Selection Networks with Spatially Random Relays
}

\author{
Yu Gong, Gaojie Chen, Member, IEEE, Tian Xie
}

\begin{abstract}
It is well recognized that using buffers in relay networks significantly improves the transmission reliability, which is often at the price of higher packet delay. Existing buffer-aided relay networks are all based on the physical links among cooperative nodes. This may however lead to performance degradation in practice, because that cooperative nodes may not trust each other for cooperation even though their physical connection are strong. In this paper, we propose a novel buffer-aided relay selection scheme to align data transmission with both strong and trusted links. By maintaining the buffer lengths as close as possible to the newly introduced target buffer lengths, the proposed scheme is able to balance the outage performance and packet delay. Both the outage probability and average packet delay are analyzed for spatially random relays. Particularly we show that outage performance may have error floors because of the trusts. The analysis shows that using buffers in trust aware relay networks is able to either increase the diversity order or lower the error floor of the outage probability.
\end{abstract}

Index Terms-Social trusts, buffer-added relay selection, stochastic geometry

\section{INTRODUCTION}

In traditional relay selection schemes, relays with strong physical links are selected for cooperative transmission. Because relays are mobile devices which are often handled by human users, the selected relay may not always be willing to help the transmission source. For example, a person is likely to use his/her mobile to help data transmission for his/her friends but not for strangers. Therefore, the effectiveness of cooperative transmission depends on not only physical links but also trusts among cooperative users. Exploring users' trusts in wireless networks has attracted much attention recently [1]-[3]. While various trust models (e.g. distance-based and rank-based trust models in [4], [5] respectively) have been proposed, the detail is beyond the scope of this paper. In this paper, we focus on how trusts (which are assumed to be available) are used to improve the relay selection. Social trusts can be represented either by binary values with 0 and 1 corresponding to no and full trust between two users respectively [2], or by a real value within $[0,1]$ which represents the probability that two users are in trust [6]. In either case, only when a relay is in trust with the source transmitter, is it willing to join the cooperative transmission. This paper adopts the trust model in [6] as it describes a more general scenario. In relay selection networks, the difficulty is to decide whether a relay with strong links or high trusts shall be selected. In [6], the channel gains and trusts are incorporated into a single signal-to-noise-ratio (SNR) measurement and the relay with the maximum SNR is selected for cooperative transmission. While the selected relay can compromise between the channel gains and trusts, the full potential of the relay network is not fully released.

Y. Gong and T. Xie are with the Wolfson School of Mechanical, Electrical and Manufacturing Engineering, Loughborough University, Loughborough, Leicestershire, UK, Email: y. gong@lboro.ac.uk.

G. Chen is with the Department of Engineering, University of Leicester, Leicester, UK, Emails: gaojie.chen@leicester.ac.uk.
This is because both channel gains and trusts are uncorrelated random values. It is thus often that the selected relay is not in trust with the source when the corresponding links are strong, or the links are weak when the selected relay is in trust with the source.

On the other hand, buffer-aided schemes have attracted much recent attention in many research topics, i.e., cognitive radio networks [7] and physical layer security [8]. In [9], [10], novel buffer-aided adaptive link schemes have been proposed for 3node relay networks, and the work was extended to bidirectional 3 -node relay work in [11], [12]. Buffer-aided schemes can also work with space-time coding and network coding as is described in [13] and [14] respectively. In buffer-aided approaches, relays are able to store the data in buffers and hold the transmission until the corresponding links are strong enough, making it flexible for the cooperative transmission to align with 'strong' links. This encourages us to apply the buffers in trust aware relay networks, where the purpose is to better synchronize the transmission with both strong and trusted links. While various buffer-aided relay selection schemes have been proposed (e.g. [15], [16]), there is still large room for further improvement and none of them considers the trusts. Compared with their non-buffer aided counterparts, all buffer-aided schemes have coding gains which is $3 \mathrm{~dB}$ for independent identical distributed (i.i.d.) fading channels [17], but not all of them have diversity gains. In principle, the diversity order of a relay selection system depends on the number of available links for selection. For a relay network with $K$ relays, there are $2 K$ links in total, but not all of them are always available for selection. To be specific, when the buffer of a relay becomes full or empty, the corresponding source-to-relay or relay-to-destination link is not available for selection respectively and so the diversity order is decreased by one. The max-link relay selection can achieve full diversity order of $2 K$ but only when the buffer sizes are infinite and all channels are i.i.d. fading [16]. In practice, however, because the buffer sizes are always limited and/or the channels may not be i.i.d., the diversity order is often well below $2 K$. When the trust is considered, even with i.i.d channels and infinite buffer sizes, the max-link can still not achieve full diversity. Because relay users often have different trusts with the source node, the probabilities to select different links are not the same (even for i.i.d. channels). This makes buffers more likely be empty or saturate, leading to diversity order lost.

Although applying buffers brings in coding and/or diversity gain, it also increases the packet delay because of the packet queuing at the buffers. In order to minimize the packet delay, the queues at the buffers shall be maintained as short as possible. This however contradicts the requirements for the diversity performance where the buffers shall avoid from being empty (or full). In [17], we proposed a delay reduced buffer-aided scheme, in which higher selection priority is given to relay-to-destination than to source-to- 
relay links, so data is moving out of buffers as quickly as possible to minimize the queuing lengths. While the proposed scheme in [17] significantly reduces the packet delay, it only has coding gain but little diversity gain.

In this paper, we propose a novel trust aware buffer-aided relay selection scheme which is able to balance the diversity gain and packet delay. This is achieved by introducing a new set of parameters, the 'target-queuing-lengths', from which the selection priorities are given to all available links. To be specific, by choosing appropriate target-queuing-lengths, the proposed scheme can be tuned to achieve minimum packet delay or maximum diversity order, or a compromise between the diversity and delay according to system requirements. The contribution of this paper is summarized as following:

- Propose a novel buffer-aided scheme which can balance the diversity and packet delay according to system requirements, where both physical links and trusts are considered (in Section III).

- Analyze the outage probability of the proposed scheme based on the random positions of the relays (in Section IV).

- Derive the average packet delay, diversity order and error floor of the proposed scheme (in Section V).

- Derive the optimum target buffer lengths for best outage performance and minimum average packet delay respectively (in Section VI).

\section{System MODEL}

The system model of the buffer-aided relay network is shown in Fig. 1, where there are one source node $S$, one destination node $D$ and $K$ relay nodes $R_{k}$ for $k=1, \cdots, K$. We assume that relays are randomly positioned in the circular area $A^{1}$, where the centre of $A$ is in the middle of the line connecting $S$ and $D$, and the radius is $r_{o}$. Every relay is equipped with a data buffer of finite size $L$, and applies decode-and-forward (DF) protocol. We assume relay positions follow binomial position process (BPP). The BPP is often used to describe a group of fixed numbered nodes' random positions within an area [18], [19].

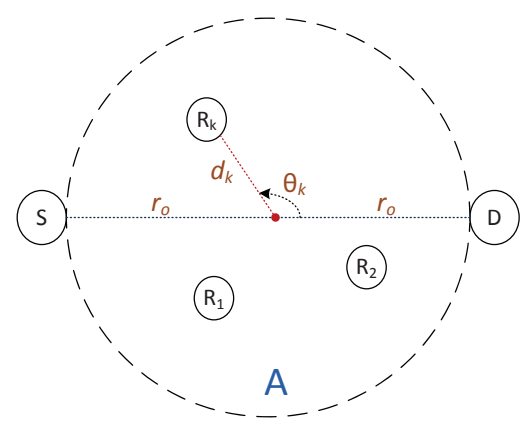

Fig. 1. System model for a relay network with one source, one destination and $K$ relays randomly positioned in the area $A$.

In this paper, we assume the channels are independent and non identically distributed (i.n.i.d.) Rayleigh fading channels ${ }^{2}$. Thus the

\footnotetext{
${ }^{1}$ The "'circular area' assumption is for better exposition of the performance analysis, which is not required to apply the proposed scheme in practical systems.

${ }^{2}$ The Rayleigh fading assumption is for clear analysis and fair comparison with existing relay selection schemes since most of them also apply the same assumption. When other channel fading scenario such as the shadowing effect is considered, the proposed scheme can still be applied. Although the analytical expressions will be different from those for the Rayleigh fading, the improvement of the proposed scheme compared with existing schemes can still be similarly obtained.
}

SNR of $S \rightarrow R_{k}$ and $R_{k} \rightarrow D$ links are given by

$$
\gamma_{s, r_{k}}=\frac{P_{t x}\left|h_{s, r_{k}}\right|^{2}}{\sigma_{n}^{2} d_{s, r_{k}}^{\alpha}}=\frac{g_{s, r_{k}}}{d_{s, r_{k}}^{\alpha}}, \quad \gamma_{r_{k}, d}=\frac{P_{t x}\left|h_{r_{k}, d}\right|^{2}}{\sigma_{n}^{2} d_{r_{k}, d}^{\alpha}}=\frac{g_{r_{k}, d}}{d_{r_{k}, d}^{\alpha}},
$$

respectively, where $h_{s, r_{k}}$ and $h_{r_{k}, d}$ are the Rayleigh fading coefficients, $d_{s, r_{k}}$ and $d_{r_{k}, d}$ are the distances, $\alpha$ is the path loss factor, $g_{s, r_{k}}=P_{t x}\left|h_{s, r_{k}}\right|^{2}, g_{r_{k}, d}=P_{t x}\left|h_{r_{k}, d}\right|^{2}$, and $\sigma_{n}^{2}$ denotes the power of complex additive white Gaussian noise (AWGN) and $P_{t x}$ is the transmission power which is assumed to be the same at every transmission node. We assume without losing generality that all noise powers are normalized to unit.

We assume Rayleigh fading gains $\left|h_{s r_{k}}\right|^{2}$ and $\left|h_{r_{k}, d}\right|^{2}$ are normalized i.i.d. exponentially distributed. Thus $g_{s, r_{k}}$ and $g_{r_{k}, d}$ are also i.i.d. exponentially distributed with the average gain as $\bar{\lambda}=P_{t x}$. As is illustrated in Fig. 1 , the position of relay $R_{k}$ is represented as $\left(d_{k}, \theta_{k}\right)$, and we have

$$
\begin{aligned}
& d_{s, r_{k}}=\sqrt{r_{o}^{2}+d_{k}^{2}-2 r_{o} d_{k} \cos \left(\pi-\theta_{k}\right)} \\
& d_{r_{k}, d}=\sqrt{r_{o}^{2}+d_{k}^{2}-2 r_{o} d_{k} \cos \left(\theta_{k}\right)}
\end{aligned}
$$

It is clear from (2) that both $d_{s, r_{k}}$ and $d_{s, r_{k}}$ are functions of $d_{k}$ and $\theta_{k}$. Thus if two links correspond to the same relay node, their path losses are dependently distributed, so are their overall channel gains; otherwise, their path loss and overall channel gain distributions are independent. In both cases, because the Rayleigh fading gains are i.i.d. and positions of all relay nodes follow binomial position process, the overall channel gain distributions are all identical. We note that, if the relay nodes' position are fixed, all channel gains become independent, but they are usually not identically distributed as the relay nodes often have different distances to the source and destination nodes.

The trust level between the source $S$ and relay $R_{k}$ is represented by the weight $P_{\varphi_{k}}$, where $P_{\varphi_{k}}=P\left(\varphi_{k}(t)=1\right)$ and $\varphi_{k}(t)$ is a binary parameter to indicate whether $S$ and $R_{k}$ are in trust at time $t$ or not. At time $t$, only if $\varphi_{k}(t)=1$, will $R_{k}$ receive the data from $S$, otherwise $R_{k}$ refuses to receive. Once the relay $R_{k}$ receives one data packet from $S$ and stores it in its buffer, it will always forward it to the destination at later time.

\section{SELECTION RULE}

At any time, the numbers of data packets in all relay buffers form a "state". If the relay number is $K$ and buffer size is $L$, there are $(L+1)^{K}$ states in total. The $i$-th state vector is defined as

$$
\mathbf{q}_{i}=\left[q_{i, 1}, \cdots, q_{i, K}\right], \quad i=1, \cdots,(L+1)^{K},
$$

where $q_{i, k}$ is the buffer queuing length at $R_{k}$ at state $\mathbf{q}_{i}$. On the one hand, buffers shall avoid from being empty or full to achieve high diversity order; On the other hand, keeping buffers empty helps to minimize the packet delays. In order to trade off the diversity and delay performance, we introduce the target buffer length (denoted as $\Theta_{k}$ ) at every relay node, and maintain the buffer queuing length as close to $\Theta_{k}$ as possible. This is achieved by giving higher priorities to links corresponding to buffer lengths further away from their respective target lengths. At any buffer state, once the priority orders for selection for all available are obtained, the proposed link selection rules are applied as following:

- Consider the link with highest priority first, or if multiple links have the same priority, consider the link with the highest SNR.

- If this link supports the target transmission rate and the corresponding relay and source are in trust (i.e. $\varphi_{k}(t)=1$ ), select this link. 
- Otherwise consider the $\operatorname{link}(\mathrm{s})$ with the next highest priority, and repeat this process until one link is selected, or outage occurs if no link can be selected.

Below we first give priority orders to all relay nodes, from which the priorities for all links are obtained. Supposing the buffer state is $\mathbf{q}_{i}$, the difference between target length and the corresponding buffer length is given by $\Delta_{k}=q_{i, k}-\Theta_{k}$. Then higher priority is given to relays with larger $\Delta_{k}$. Particularly if $\Delta_{x}=\Delta_{y}$, relays $R_{x}$ and $R_{y}$ have the same priority. But if $\Delta_{x}=-\Delta_{y}>0, R_{x}$ is given higher priority order than $R_{y}$. This is because, while the buffer queuing lengths at $R_{x}$ and $R_{y}$ are at the same distance away from $\Theta_{k}$, selecting $R_{x}$ corresponds to reducing the buffer length, which again leads to lower delay.

From the above, we obtain the priority order of $R_{k}$ which is denoted as $\mathcal{O}\left(R_{k}\right)$, where the $m$-th highest priority corresponds to $\mathcal{O}()=$.$m . Every relay R_{k}$ corresponds a pair of $S \rightarrow R_{k}$ and $R_{k} \rightarrow D$ links. At state $\mathbf{q}_{i}$, if only one of the pair of links is available, the priority order for this link is the same as that for the relay which is $\mathcal{O}\left(R_{k}\right)$. Otherwise, if both links are available, we have the following 2 cases:

- Case 1: $\Delta\left(R_{k}\right) \geq 0$. In this case, because the buffer length is longer than the target length $\Theta_{k}$, it is preferable to move data out of buffer (rather than moving into it). Thus the priority order of the $R_{k} \rightarrow D$ link is set as same as that for the relay node which is $\mathcal{O}\left(R_{k}\right)$, and the priority order for the $S \rightarrow R_{k}$ link is set to the $\mathcal{O}\left(R_{k}\right)$-th from the last.

- Case 2: $\Delta\left(R_{k}\right)<0$. Similarly, the priority order of the $S \rightarrow$ $R_{k}$ link is set to $\mathcal{O}\left(R_{k}\right)$, and the priority order for the $R_{k} \rightarrow$ $D$ link is the $\mathcal{O}\left(R_{k}\right)$ from the last.

A central control node, which can be either an independent node or among existing nodes, is used to make the selection decision. The central node requires the knowledge of all links' channel gains and trust values, but not the knowledge of the buffer states. This is because buffers can always start from empty and the central node can monitor the buffer states. Thus the implementation of the proposed scheme requires the same knowledge as most other relay selection schemes including the non-buffer-aided max-min scheme and the existing buffer-aided max-max [15] and max-link [16]) schemes.

Before leaving this section, Fig. 2 shows an example to set the link priority orders in a 5-relay-nodes network, where the buffer size is $L=4$, the target buffer lengths are $\Theta_{k}=2$, and the buffer state is $\mathbf{q}_{i}=[0,2,2,3,4]$. The relays are ordered as, from highest to lowest priority, $R_{5}, R_{1}, R_{4}, R_{2}$ and $R_{3}$ respectively. There are 8 available links and the priority orders for every available links are also shown in Fig. 2, where $\operatorname{link}_{m}$ indicates the priority order for the corresponding link is $m$.

\section{OUTAGE PROBABILITY}

We denote $\mathbf{A}$ as the $(L+1)^{K} \times(L+1)^{K}$ state transition matrix, where the entry $A_{i j}=P\left(\mathbf{q}_{i} \mid \mathbf{q}_{j}\right)$ which is the transition probability from state $\mathbf{q}_{j}$ to $\mathbf{q}_{i}$. Particularly $A_{i i}$ is the outage probability at state $\mathbf{q}_{i}$. Below we derive the transition probability $A_{i j}$ for $i \neq j$ and $i=j$, from which we obtain the outage probability.

\section{A. Outage probability at state $\mathbf{q}_{i}: P_{\text {out }}^{\mathbf{q}_{i}}=A_{i i}$}

At state $\mathbf{q}_{i}$, the outage occurs if all available links are in outage, and thus the outage probability is given by

$$
P_{\text {out }}^{\mathbf{q}_{i}}=A_{i i}=P\left(\text { out }_{1}, \cdots, \text { out }_{N_{i}}\right)
$$

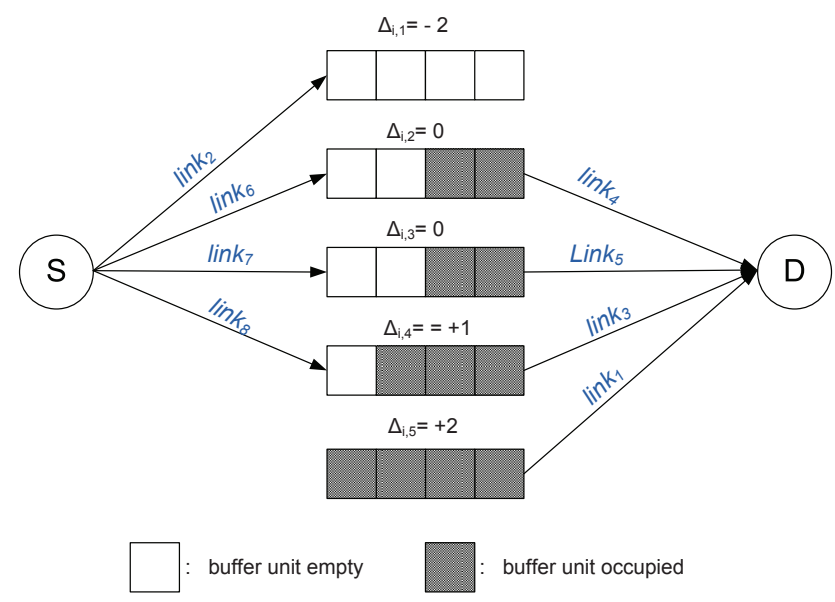

Fig. 2. An example of setting link priority orders: the target buffer length $\Theta_{k}=2$.

where $N_{i}$ is the number of available links at state $\mathbf{q}_{i}$, out $t_{m}$ is the event that $l i n k_{m}$ is in outage, and $l i n k_{m}$ is the link with the $m$-th priority order of selection. Because the channel gains for any two links are independent only if they correspond to different relay nodes, (4) can be decomposed into $K$ terms, each corresponding to one relay node. We assume without losing generality that there are $x$ relay nodes with both $S \rightarrow R_{k}$ and $R_{k} \rightarrow D$ links available, where these $x$ pair of links are denoted as $\left(\right.$ link $_{a_{1}}$, link $\left._{a_{1}^{\prime}}\right), \cdots,\left(\right.$ link $_{a_{x}}$, link $\left._{a_{x}^{\prime}}\right)$ respectively. The remaining $\left(K-x\right.$ ) relays nodes only have one of the $S \rightarrow R_{k}$ and $R_{k} \rightarrow D$ links available, denoted as $\left(\operatorname{lin}_{b_{1}}, \cdots, \operatorname{lin}_{b_{K-x}}\right)$ respectively. Thus (4) can be expressed as

$$
\begin{aligned}
& P_{\text {out }}^{\mathbf{q}_{i}}= \underbrace{P\left(\text { out }_{a_{1}}, \text { out }_{a_{1}^{\prime}}\right), \cdots, P\left(\text { out }_{a_{x}}, \text { out }_{a_{x}^{\prime}}\right)} \\
& \times \underbrace{P\left(\text { out }_{b_{1}}\right), \cdots, P\left(\text { out }_{b_{K-x}}\right)},
\end{aligned}
$$

In the following we derive $P\left(\right.$ out $\left._{m}\right)$ and $P\left(\right.$ out $_{m}$, out $\left._{m^{\prime}}\right)$.

1) $P\left(\right.$ out $\left._{m}\right)$ : From (1), the link capacity for $l i n k_{m}$ is given by $C_{m}=\log \left(1+g_{m} / d_{m}^{\alpha}\right)$, where the subindex $m$ corresponds for either a $S \rightarrow R_{k}$ or a $R_{k} \rightarrow D$ link. If $l i n k_{m}$ is a $S \rightarrow R_{k}$ link, out $t_{m}$ occurs when either $C_{m}<\eta$, or $S$ and $R_{k}$ are not in trust (i.e. $\varphi_{k}=0$ ), where $\eta$ is the target data rate. If $l i n k_{m}$ is a $R_{k} \rightarrow D$ link, out $t_{m}$ occurs only when $C_{m}<\eta$, because data will be forwarded to the destination once it is stored in a relay buffer. Thus we have

$$
P\left(\text { out }_{m}\right)=\left\{\begin{array}{l}
P_{\varphi_{k}} P\left(C_{m}<\eta\right)+\left(1-P_{\varphi_{k}}\right), \text { link }_{m} \in\left\{S \rightarrow R_{k}\right\} \\
P\left(C_{m}<\eta\right), \\
\text { link }_{m} \in\left\{R_{k} \rightarrow D\right\}
\end{array}\right.
$$

where $P\left(C_{m}<\eta\right)$ is given by

$$
\begin{aligned}
P\left(C_{m}<\eta\right) & =\int_{A} P\left(C_{m}<\eta \mid R_{k}\right) p\left(R_{k}\right) d A \\
& =\int_{A} P\left(\log \left(1+\frac{\left|h_{m}\right|^{2}}{d_{m}^{\alpha}}\right)<\eta\right) p\left(R_{k}\right) d A
\end{aligned}
$$

where $P\left(C_{m}<\eta \mid R_{k}\right)$ is the probability for $C_{m}<\eta$ conditioned on the position of $R_{k}$, and $p\left(R_{k}\right)$ is the probability density function of position of $R_{k}$. Because relays follow binomial point process, we have $p\left(R_{k}\right)=1 /\left(\pi r_{o}^{2}\right)$. Then as is illustrated in Fig. 1, we have

$$
P\left(C_{m}<\eta\right)=\frac{1}{\pi r_{o}^{2}} \int_{r=0}^{r_{o}} \int_{\theta=0}^{2 \pi}\left(1-e^{-\frac{d_{m}^{\alpha} \cdot \xi}{\lambda}}\right) r d r d \theta
$$


where $\xi=2^{\eta}-1$.

2) $P\left(\right.$ out $_{m}$, out $\left._{m^{\prime}}\right)$ : We assume without losing generality that $\operatorname{link}_{m}$ and $\operatorname{link}_{m^{\prime}}$ are the $S \rightarrow R_{k}$ and $R_{k} \rightarrow D$ links respectively. Considering both the line capacities and the trust, we have

$$
\begin{aligned}
P\left(\text { out }_{m}, \text { out }_{m^{\prime}}\right)= & P_{\varphi_{k}} P\left(C_{m}<\eta, C_{m^{\prime}}<\eta\right) \\
& +\left(1-P_{\varphi_{k}}\right) P\left(C_{m^{\prime}}<\eta\right),
\end{aligned}
$$

where $P\left(C_{m^{\prime}}<\eta\right)$ can be obtained as in (8), and

$$
\begin{aligned}
P & \left(C_{m}<\eta, C_{m^{\prime}}<\eta\right) \\
& =\int_{A} P\left(C_{m}<\eta \mid R_{k}\right) P\left(C_{m^{\prime}}<\eta \mid R_{k}\right) p\left(R_{k}\right) d A \\
& =\frac{1}{\pi r_{o}^{2}} \int_{r=0}^{r_{o}} \int_{\theta=0}^{2 \pi}\left(1-e^{-\frac{d_{m}^{\alpha} \cdot \xi}{\lambda}}\right)\left(1-e^{-\frac{d_{m^{\prime}}^{\alpha} \cdot \xi}{\lambda}}\right) r d r d \theta
\end{aligned}
$$

\section{B. Transition probability $A_{j i}=P\left(\mathbf{q}_{j} \mid \mathbf{q}_{i}, i \neq j\right)$}

Suppose if $\operatorname{link}_{n}$ (with $n$-th priority order) is selected, buffer state transits from $\mathbf{q}_{i}$ to $\mathbf{q}_{j}$. Without losing generality, we assume $\operatorname{lin} k_{n}, \operatorname{lin} k_{n+1}, \cdots \operatorname{lin} k_{n+m}$ have the same priority order. Thus $\operatorname{link}_{n}$ is selected if (1) all links with lower priority order than $\operatorname{link}_{n}$ are in outage, (2) $l i n k_{n}$ has the highest SNR among link $k_{n}, \cdots, \operatorname{lin}_{n+m}$ links, and (3) $l i n k_{n}$ is not in outage. Thus we have

$$
\begin{aligned}
A_{j i}= & P\left(\text { link }_{n} \text { is selected }\right)=P\left(\text { out }_{1}, \cdots, \text { out }_{n-1}, \overline{\text { out }}_{n}\right) \\
& \times P\left(\gamma_{n}>\max \left\{\gamma_{n+1}, \cdots, \gamma_{n+m}\right\}\right), \quad i \neq j
\end{aligned}
$$

where $\overline{o u t}_{m}$ is the events that $\operatorname{link}_{m}$ is not in outage.

We assume $l i n k_{n}$ and $l i n k_{n^{\prime}}$ associate with the same relay node. If $l i n k_{n^{\prime}}$ is not available or has lower priority order than $l i n k_{n}$, link $k_{n^{\prime}}$ does not appear in (11). Otherwise if $l i n k_{n^{\prime}}$ is available and has higher priority order than $\operatorname{lin} k_{n}$, it appears in (11). Thus we have

$$
\begin{aligned}
A_{j i}= & \underbrace{P\left(\text { out }_{a_{1}}, \text { out }_{a_{1}^{\prime}}\right), \cdots, P\left(\text { out }_{a_{x}}, \text { out }_{a_{x}^{\prime}}\right)} \\
& \times \underbrace{P\left(\text { out }_{b_{1}}\right), \cdots, P\left(\text { out }_{b_{y}}\right)} \\
& \times P\left(\gamma_{n}>\max \left\{\gamma_{n+1}, \cdots, \gamma_{n+m}\right\}\right) \cdot B, \text { for } i \neq j
\end{aligned}
$$

where $B=P\left(\right.$ out $\left._{n^{\prime}}, \overline{\text { out }}_{n}\right)$ or $B=P\left(\overline{\text { out }}_{n}\right)$ if $l i n k_{n^{\prime}}$ does or does not appear in (11) respectively, $\operatorname{lin} k_{a_{x}}$ and $\operatorname{lin} k_{a_{x}^{\prime}}$ correspond to the same relay and all indices $\left\{a_{x}, b_{y}\right\} \in\{1, \cdots, n-1\}$.

While $P\left(\overline{o u t}_{n}\right)=1-P\left(\right.$ out $\left._{n}\right), P\left(\right.$ out $\left._{m}\right)$ and $P\left(\right.$ out $_{m}$, out $\left._{m^{\prime}}\right)$ are obtained in (6) and (9) respectively. Below we drive $P\left(\right.$ out $\left._{n^{\prime}}, \overline{\text { out }}_{n}\right)$ and $P\left(\gamma_{n}>\max \left\{\gamma_{n+1}, \cdots, \gamma_{n+m}\right\}\right)$.

Following the similar procedure in deriving (6), we have

$$
P\left(\text { out }_{n^{\prime}}, \overline{\text { out }}_{n}\right)=\left\{\begin{array}{r}
P_{\varphi_{k}} P\left(C_{n}<\eta, C_{n^{\prime}}>\eta\right), \\
\text { if link } k_{n} \in\left\{S \rightarrow R_{k}\right\} \\
P_{\varphi_{k}} P\left(C_{n}<\eta, C_{n^{\prime}}>\eta\right) \\
+\left(1-P_{\varphi_{k}}\right) P\left(C_{n^{\prime}}>\eta\right), \\
\text { if } l_{i n k_{n}} \in\left\{R_{k} \rightarrow D\right\}
\end{array}\right.
$$

where $P\left(C_{n}<\eta, C_{n^{\prime}}>\eta\right)$ is obtained as

$$
\begin{aligned}
P\left(C_{n}<\eta,\right. & \left.C_{n^{\prime}}<\eta\right) \\
& =\int_{A} P\left(C_{n}<\eta \mid R_{k}\right) P\left(C_{n^{\prime}}>\eta \mid R_{k}\right) p\left(R_{k}\right) d A \\
& =\frac{1}{\pi r_{o}^{2}} \int_{r=0}^{r_{o}} \int_{\theta=0}^{2 \pi}\left(1-e^{-\frac{d_{n}^{\alpha} \cdot \xi}{\lambda}}\right) e^{-\frac{d_{n^{\prime} \cdot \xi}^{\alpha}}{\lambda}} r d r d \theta
\end{aligned}
$$

We have shown in Section II that channel gains are identical distributed. Furthermore, if multiple links have the same priority order, they must not correspond to the same relay node and thus are also independent. Therefore, all links with the same priority order must have i.i.d. channel gains, and we have

$$
P\left(\gamma_{n}>\max \left\{\gamma_{n+1}, \cdots, \gamma_{n+m}\right\}=\frac{1}{m+1}\right.
$$

Finally the outage probability of the overall system is given by

$$
P_{\text {out }}=\sum_{i=1}^{(L+1)^{N}} \pi_{i} \cdot p_{\text {out }}^{\mathbf{q}_{i}},
$$

where $\pi_{i}$ is the stationary probability for state $\mathbf{q}_{i}$. Because the transition matrix $\mathbf{A}$ is column stochastic, irreducible and aperiodic, the stationary state probability vector is obtained as (see [20], [21]) $\boldsymbol{\pi}=(\mathbf{A}-\mathbf{I}+\mathbf{B})^{-1} \mathbf{b}$ where $\boldsymbol{\pi}=\left[\pi_{1}, \cdots, \pi_{(L+1)^{N}}\right]^{\mathrm{T}}$, $\mathbf{b}=(1,1, \ldots, 1)^{T}, \mathbf{I}$ and $\mathbf{B}$ are the identity and all one matrices with appropriate dimensions respectively. Substituting it into (16) gives the outage probability as

$$
P_{\text {out }}=\operatorname{diag}(\mathbf{A})^{\mathrm{T}} \cdot(\mathbf{A}-\mathbf{I}+\mathbf{B})^{-1} \mathbf{b},
$$

where $\operatorname{diag}(\mathbf{A})$ is a vector consisting of all diagonal elements of $\mathbf{A}$, and $A_{j i}$ is obtained in (5) and (12) for $i=j$ and $i \neq j$ respectively.

\section{DELAY AND DIVERSITY PERFORMANCE}

This section derives the average packet delay, diversity order and error floor to better reveals the performance of the proposed scheme.

\section{A. Average packet delay}

The delay of a packet in the system is the number of time slots for a packet to leave the source node and arrive the destination. Because it takes one time slot to transmit a packet from the source to a relay node, the average packet delay in the system is given by

$$
\bar{D}=1+\bar{D}_{r}
$$

where $\bar{D}_{r}$ is the average delay at the relay nodes. Based on Little's Law [22], we have

$$
\bar{D}_{r}=\frac{1}{\bar{\xi}} \sum_{k=1}^{K} \bar{q}_{k},
$$

where $\bar{\xi}$ is the average throughput of the network and $\bar{q}_{k}$ is the average buffer queuing length at relay $R_{k}$.

Because every packet takes two time slots (not necessarily consecutively) to reach the destination, the average data rate (without considering the outage probability) is $1 / 2$ data packet per time slot. Thus we have $\bar{\xi}=1 / 2 \cdot\left(1-P_{\text {out }}\right)$. On the other hand, $\bar{q}_{i}$ is obtained by averaging the buffer lengths at $R_{k}$ over all states $\mathbf{q}_{i}$ as

$$
\bar{q}_{k}=\sum_{i=1}^{(L+1)^{N}} \pi_{i} q_{i, k} .
$$

Substituting $\bar{\eta}=1 / 2 \cdot\left(1-P_{\text {out }}\right)$ and (20) into (18), gives the average packet delay in the proposed scheme as

$$
\bar{D}=1+\frac{2}{1-P_{\text {out }}} \sum_{k=1}^{K} \sum_{i=1}^{(L+1)^{N}} \pi_{i} q_{i, k} .
$$

\section{B. Diversity order}

The diversity order is defined as

$$
d=-\lim _{\bar{\lambda} \rightarrow \infty} \frac{\log P_{\text {out }}}{\log \bar{\lambda}} .
$$


The system's diversity order is determined by the individual diversity order at every state, and the general closed form is difficult (if not impossible) to obtain. Below we derive the diversity order at state $\mathbf{q}_{i}$ and in the next section we derive the overall system diversity order for optimum target buffer lengths.

The diversity order at $\mathbf{q}_{i}$ is the contribution from all relay nodes. Specifically, the diversity order contributed from relay $R_{k}$ consists of 3 cases:

1) Case 1 - Both $S \rightarrow R_{k}$ and $R_{k} \rightarrow D$ links are available at $R_{k}$ : This corresponds to a ' $P\left(\right.$ out $_{m}$, out $\left._{m^{\prime}}\right)$ ' outage term in (5). Using (9) into (22), and from (8) and (10), the diversity order contributed from relay $R_{k}$ in this case is given by

$$
\begin{aligned}
d_{s \rightarrow r_{k} \rightarrow d} & =-\lim _{\bar{\lambda} \rightarrow \infty} \frac{\log \left(P\left(\text { out }_{m}, \text { out }_{m^{\prime}}\right)\right)}{\log \bar{\lambda}} \\
& =-\lim _{\bar{\lambda} \rightarrow \infty} \frac{\log \left(\bar{\lambda}^{-2} \cdot P_{\varphi_{k}} F_{2}+\bar{\lambda}^{-1} \cdot\left(1-P_{\varphi_{k}}\right) F_{1}\right)}{\log \bar{\lambda}} \\
& = \begin{cases}1, & P_{\phi_{k}}<1, \\
2, & P_{\phi_{k}}=1\end{cases}
\end{aligned}
$$

where $\quad F_{1}=1 / \pi \int_{r=0}^{r_{o}} \int_{\theta=0}^{2 \pi} d_{m}^{\alpha} d_{m^{\prime}}^{\alpha} \xi r d r d \theta, \quad F_{2}=$ $1 / \pi \int_{r=0}^{r_{o}} \int_{\theta=0}^{2 \pi} d_{m}^{\alpha} \xi r d r d \theta$. It is interesting to observe that, if $R_{k}$ and $S$ are in full trust (i.e. $P_{\phi_{k}}=1$ ), although $S \rightarrow R_{k}$ and $R_{k} \rightarrow D$ links have dependent channel gains, they still contribute 2 diversity orders.

2) Case 2 - only $S \rightarrow R_{k}$ link is available at $R_{k}$ : It corresponds to a ' $P\left(\right.$ out $\left._{m}\right)$ ' outage term in (5). From (6), the diversity order contributed by $R_{k}$ is obtained as

$$
\begin{aligned}
d_{s \rightarrow r_{k}} & =-\lim _{\bar{\lambda} \rightarrow \infty} \frac{\log P_{\phi_{k}} P\left(C_{m}<\eta\right)+\left(1-P_{\phi_{k}}\right)}{\log \bar{\lambda}} \\
& = \begin{cases}0, & P_{\phi_{k}}<1, \\
1, & P_{\phi_{k}}=1\end{cases}
\end{aligned}
$$

3) Case 3 - only $R_{k} \rightarrow D$ link is available at $R_{k}$ : The diversity order contributed by $R_{k}$ is given by

$$
d_{r_{k} \rightarrow d}=-\lim _{\bar{\lambda} \rightarrow \infty} \frac{\log \left(P\left(\text { out }_{m}\right)\right)}{\log \bar{\lambda}}=-\lim _{\bar{\lambda} \rightarrow \infty} \frac{\log \left(-\bar{\lambda} \cdot F_{2}\right)}{\log \bar{\lambda}}=1 .
$$

The above analysis shows that, if a relay node does not have full trust with the source node, the corresponding $S \rightarrow R_{k}$ link does not contribute to the diversity gain even if it is available. This is because when $P_{\phi_{k}}<1$, no matter how large the SNR is increased, the link outage probability for $S \rightarrow R_{k}$ never goes to zero.

Applying the above analysis in Case 1-3 gives the diversity order contributed from every node, and adding them together gives the diversity order at state $\mathbf{q}_{i}$.

\section{Error Floor}

At state $\mathbf{q}_{i}$, if there exists at least one available $R_{k} \rightarrow D$ link, its corresponding outage must have $\lim _{\bar{\lambda} \rightarrow \infty} P_{\text {out }}^{\mathbf{q}_{i}}=0$. Only at all zero state, is there no available $R_{k} \rightarrow D$ link. Thus from (16), we have

$$
\lim _{\bar{\lambda} \rightarrow \infty} P_{\text {out }}=P_{\infty}(\mathbf{q}=\mathbf{0}) \lim _{\bar{\lambda} \rightarrow \infty} P_{\text {out }}^{\mathbf{q}=\mathbf{0}}=\beta P_{\infty}(\mathbf{q}=\mathbf{0}),
$$

where $\beta=\prod_{k=1}^{K}\left(1-P_{\psi_{k}}\right)$ and $P_{\infty}(\mathbf{q}=\mathbf{0})$ is the probability that the state is all zero when SNR goes to infinity.

It is clear from (26) that if the system contains the all zero state (i.e. $P_{\infty}(\mathbf{q}=\mathbf{0}) \neq 0$ ) and no relays has full trust with the source (i.e. $P_{\psi_{k}} \neq 1$ ), we have $\lim _{\bar{\lambda} \rightarrow \infty} P_{\text {out }} \neq 0$ which is the error floor. Correspondingly the diversity order of the overall system is zero. Therefore with trusts, the outage performance depends on not only diversity order but also error floor.

\section{OPTIMUM TARGET LENGTH}

Carefully choosing target buffer lengths can adjust the buffer states, from which the outage probability and average packet delay are tuned to satisfy the requirements for various applications. In this section, we derive the optimum target buffer lengths for best outage performance and minimum average packet delay when the SNR goes to infinity. For better exposition, we assume that the buffer size $L \geq 3$ in this section. The analysis for $L<3$ can be similarly obtained.

\section{A. For best outage performance}

In order to have best outage performance, either the diversity order shall be as large as possible, or the error floor shall be as low as possible. Thus the target lengths shall at least not to be set to zero, i.e. $\Theta_{k} \neq 0$. Supposing that there exist $M$ relay nodes having full trusts with the source, there are 2 cases as following:

1) Case 1: $M \neq 0$, i.e. at least one relay node has full trust: For $\Theta_{k} \neq 0$, the buffer length at every relay can only be either $\Theta_{k}$ or $\Theta_{k}-1$. This is because, when the buffer length for relay $R_{k}$ is at the target length (i.e. $q_{i, k}=\Theta_{k}$ ), the $S \rightarrow R_{k}$ link has lower priority for selection than the $R_{k} \rightarrow D$ link, so that the buffer length $q_{i, k}$ can only be decreased by one or remain unchanged at the next time; On the other hand, when $q_{i, k}=\Theta_{k}-1$, the corresponding $R_{k} \rightarrow D$ link has lower priority than the $S \rightarrow R_{k}$ links with full trust $P_{\phi}=1$, so that $q_{i, k}$ cannot be further decreased.

Therefore as long as the target lengths for all relays are set to $2 \leq \Theta_{k} \leq L-1$, all $S \rightarrow R_{k}$ and $R \rightarrow D$ links are available at all states and so the maximum diversity order can be achieved. It is then clear that the optimum target buffer length for all relay nodes shall be set to

$$
\Theta_{o p t, k}=2, \quad k=1, \cdots, K \quad \exists P_{\phi_{k}}=1
$$

From the diversity order analysis in Section V-B, the diversity orders at all states are the same which is also the diversity order of the overall system as

$$
d=K+M, \quad \exists P_{\phi_{k}}=1
$$

It is interesting from (28) that, when all relays have full trust with the source, the proposed scheme can obtain full diversity of $2 K$, which (unlike the max-link scheme) does not require the buffersizes go to infinity.

When the optimum target buffer length is set to $\Theta_{o p t, k}=2$ for all $k$, the buffer queuing lengths can only be 1 and 2 . Thus the average buffer length at every node satisfies $1<\bar{q}_{k}<2$. Then from (21), and further noting that $\lim _{\bar{\lambda} \rightarrow \infty} P_{\text {out }}=0$, the average packet delay is given by

$$
1+2 K<\left(\lim _{\bar{\lambda} \rightarrow \infty} \bar{D}=1+2 \sum_{k=1}^{K} \bar{Q}_{k}\right)<1+4 K,
$$

where $\Theta_{k}=2, \exists P_{\phi_{k}}=1$. It is clear from (29) that the average packet delay does not depend on the buffer size, and so it is significantly smaller than that for the max-link scheme ( [23]) which is $K L+1$ when buffer size is long enough. Therefore, the proposed scheme can achieve not only better outage performance but also lower average packet delay than the max-link scheme.

2) Case 2: $M=0$, i.e. no relay node has full trust: Because no relay has full trust with the source, the buffer queuing lengths at every relay node satisfy $0 \leq q_{i, k} \leq \Theta_{k}$. This implies that, no matter how we choose the target buffer lengths, the buffers must 
consist of the all zero state. Then from the analysis Section V-C, the diversity order is zero. In order to minimize the error floor, from (26), the target buffer length shall be as large as possible to minimize $P_{\infty}(\mathbf{s}=\mathbf{0})$. Thus the optimum target buffer length is the same as the buffer size which is given by

$$
\Theta_{o p t, k}=L, \quad P_{\phi_{k}}<1 \text { for all } k
$$

\section{B. For minimum packet delay}

In order to achieve the minimum packet delay, it is clear the target buffer length shall be set to zero for all relay nodes (i.e. $\left.\Theta_{k}=0\right)$. Below we derive the diversity order, error floor and average packet delay respectively.

If the target length $\Theta_{k}=0$ for all $k$, the buffer states can only be $\mathbf{0}$ or $\mathbf{0}^{(+1)}$ when the SNR goes to infinity, where $\mathbf{0}^{(+1)}$ is the vector where one of its elements is 1 and all others are 0 . This is because when the buffer state is 0 , either one $S \rightarrow R_{k}$ link is selected that the buffer state becomes $0^{(+1)}$, or no link can be selected so that the buffer state remains at $\mathbf{0}$. On the other hand, if the buffer state is $\mathbf{0}^{(+1)}$, the $R_{k} \rightarrow D$ link corresponding to relay with buffer length 1 will be selected and the buffer state goes back to $\mathbf{0}$.

Then from the diversity order analysis in Section V-B we have

$$
\begin{cases}d=0, & P_{\phi_{k}}<1 \text { for all } k \\ M \leq d \leq M+1, & \exists P_{\phi_{k}}=1\end{cases}
$$

where $M>0$ which is the number of relays having full trusts with the source. It is clear from (31) that the diversity order depends on the number of relays with full trust with the source. Particularly, if no relay has full trust, the diversity order is 0. And from (26), the error floor is given by

$$
\begin{aligned}
\text { error floor } & =\beta P_{\infty}(\mathbf{s}=\mathbf{0}) \\
& =\frac{\beta}{2-\beta}, \quad \Theta_{k}=0 \text { and } P_{\phi_{k}}<1 \text { for all } k,
\end{aligned}
$$

where $P_{\infty}(\mathbf{s}=\mathbf{0})=1 /(2-\beta)$ which is obtained in (35) in Appendix I.

In all cases, the average packet delay is given by

$$
\lim _{\bar{\lambda} \rightarrow \infty} \bar{D}=2, \quad \Theta_{k}=0 \text { for all } k .
$$

The proof is given in Appendix I. It is clear from (33) that setting the target buffer length as $\Theta=0$ can reach the minimum possible delay when the SNR is high enough.

\section{Numerical Simulations}

In the simulations below, the target transmission rates in all schemes are set to $R=1 \mathrm{bps} / \mathrm{Hz}$. There are 3 relay nodes with positions randomly distributed within the circle shown in Fig. 1, where the circle radius is set to $r_{o}=50$. The path lose degradation factor is set to $\alpha=3$. All simulation results are obtained with 1,000,000 Monte Carlo runs. The buffer size is set to $L=5$.

We consider two cases in this section. In Case 1, one of the relays has full trust with the source such that the trust levels for the 3 relays are set to $P_{\varphi_{1}}=1, P_{\varphi_{2}}=0.8$ and $P_{\varphi_{3}}=0.6$ respectively. Fig. 3 (a) and (b) show the outage probabilities and average packet delays $v s$ transmission $\operatorname{SNR}\left(P_{t x} / \sigma_{n}^{2}\right)$ respectively. For comparison, the results for the non-buffer-aided max-min and the existing buffer-aided max-link schemes are also shown. The proposed trust-aware scheme is tuned to achieve best outage performance and minimum average packet delay by setting the target buffer length as 2 and 0 respectively. It is clearly shown that

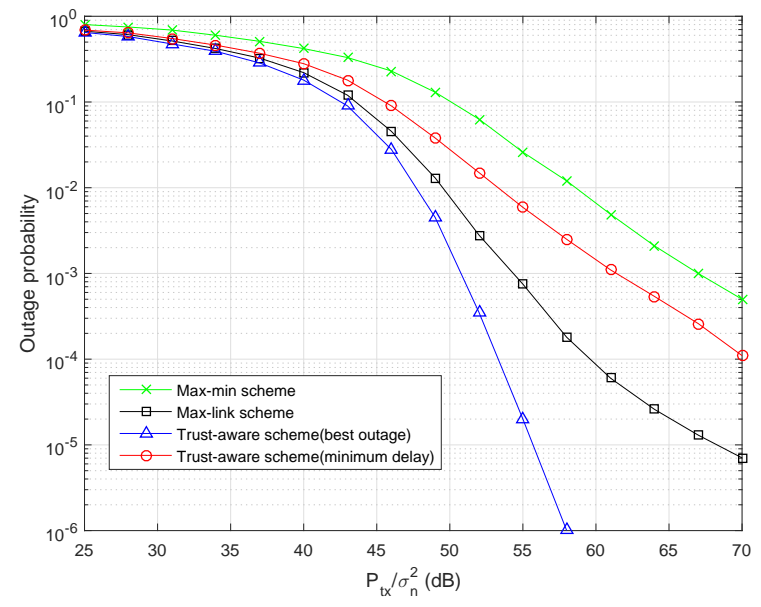

(a) Outage probability

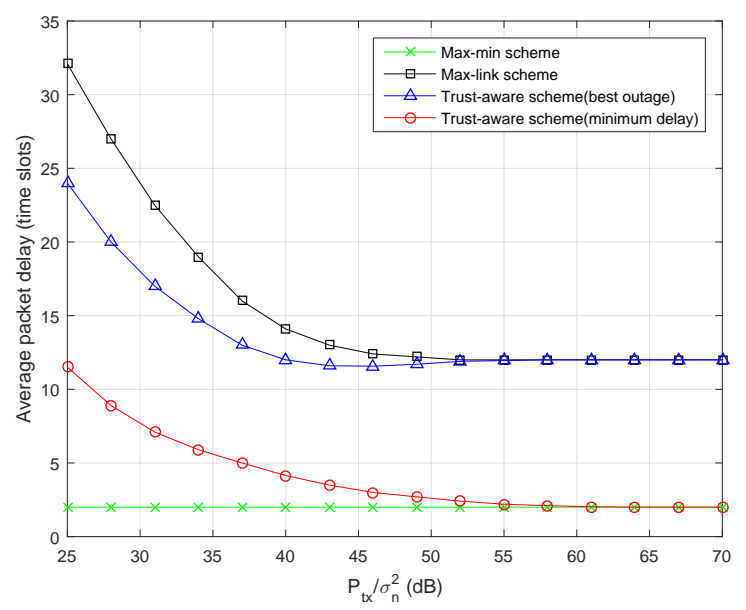

(b) Average delay

Fig. 3. Case 1 - The first relay has full trust with the source, where the trust levels are set as $P_{\varphi_{1}}=1, P_{\varphi_{2}}=0.8$ and $P_{\varphi_{3}}=0.6$ respectively. The 'Trust-aware (best outage)' and 'Trust-aware (minimum delay)' curves are for the proposed trust-aware scheme by setting target length as 2 and 0 respectively.

the trust-aware scheme for best outage is superior to the max-link scheme in both outage probability and average packet delay. On the other hand, the trust-aware scheme for minimum delay can achieve the minimum average delay of 2 when the SNR is large enough, but its outage performance is not as good as the max-link scheme.

In Case 2, no relay has full trust with the source, where the trust values are set to $P_{\varphi_{1}}=0.4, P_{\varphi_{2}}=0.3$ and $P_{\varphi_{3}}=0.2$. Fig. 4 (a) and (b) show the outage probabilities and average packet delay respectively. As is expected, the error-floors are clearly shown in Fig. 4 (a). Similar to the results in Fig. 3, the proposed trust-aware scheme can be tuned to achieve either best outage performance or minimum packet delay by setting the target buffer length as 5 and 0 respectively. Particularly the outage improvement is even more obvious in Fig. 4 than that in Fig. 3 when the proposed scheme set to achieve best outage performance. This is not surprising as the trust levels in Fig. 4 are poorer than those in Fig. 3, which makes the transmission links between $S \rightarrow R_{k}$ and $S \rightarrow D$ be more 'unbalanced'.

Fig. 5 (a) and (b) show how the outage probability and average 


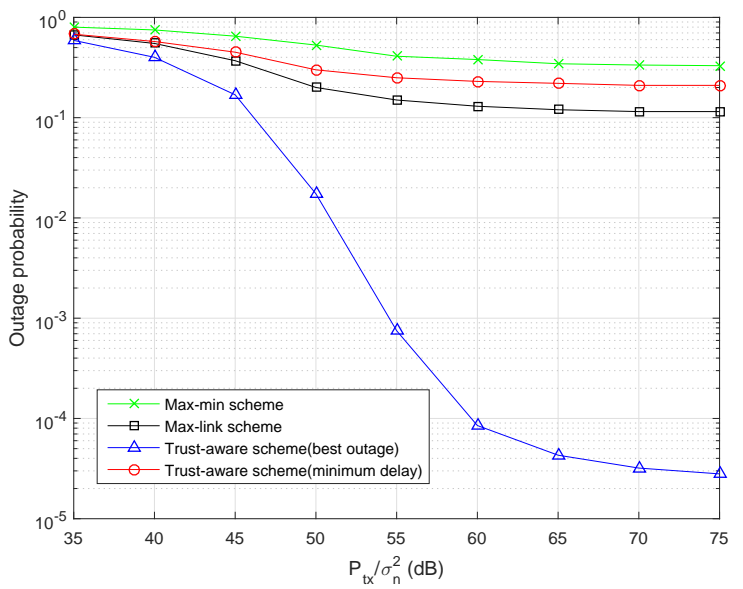

(a) Outage probability

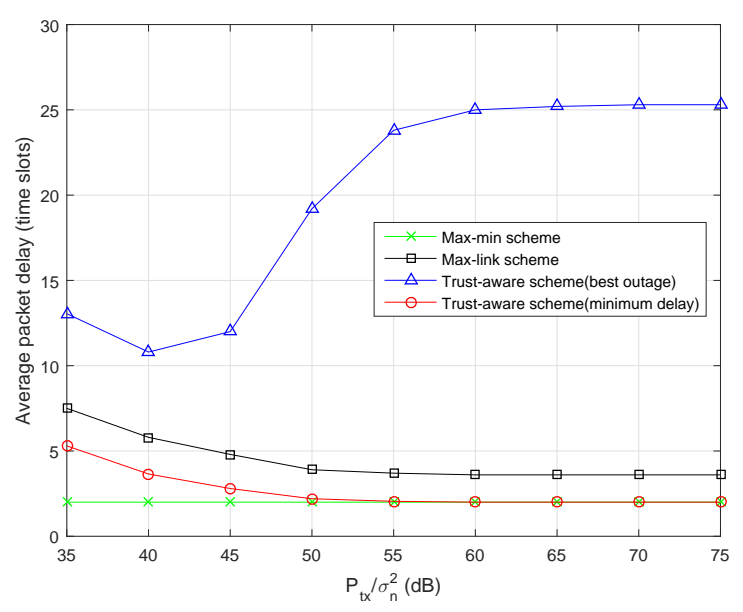

(b) Average delay

Fig. 4. Case 2 - no relay has full trust with the source, where $P_{\varphi_{1}}=0.4$, $P_{\varphi_{2}}=0.3$ and $P_{\varphi_{3}}=0.2$. The 'Trust-aware (best outage)' and 'Trustaware (minimum delay)' curves are for the proposed trust-aware scheme by setting target length as 5 and 0 respectively.

packet delay are affected by the target buffer length, where the SNR-s are fixed at $55 \mathrm{~dB}$ and $75 \mathrm{~dB}$, and all other parameters are set as same as those in Fig. 3 and 4, for Case 1 and 2 respectively. In order to observe the influence of the individual target buffer length for every relay node, Relay 1's target buffer length varies from 0 to $L$, while buffer lengths for other relays are all fixed at 2 and 5 in Case 1 and 2 respectively. It is clearly shown in Fig. 5 (a) and (b) that, in Case 1, the optimum target buffer lengths for best outage performance and minimum delay are 2 and 0 respectively. While in Case 2, the optimum target buffer length for best outage performance and minimum delay is 5 and 0 respectively. These results very well matches our analysis in Section VI.

\section{CONCLUSIONS}

This paper proposed a novel buffer-aided relay selection scheme for trust aware relay networks. By setting appropriate target buffer lengths at relay buffers, the proposed scheme is able to achieve tradeoff between the outage performance and packet delay. Particularly the proposed scheme can achieve better performance in both outage probability and average packet delay than the benchmark max-link buffer-aided scheme. Both the outage probability and

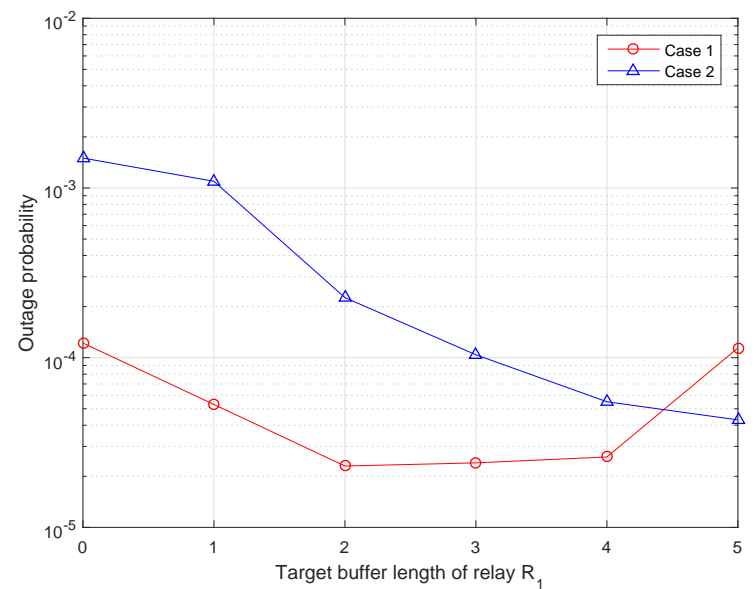

(a) Outage probability

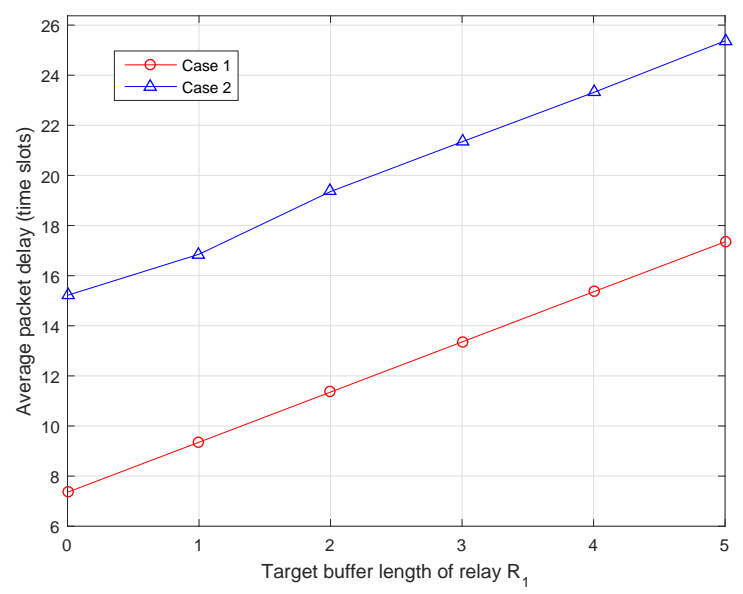

(b) Average delay

Fig. 5. Outage and delay $v s$ target buffer length for Relay 1. In Case 1: $P_{\phi_{1}}=1, P_{\phi_{2}}=0.8$ and $P_{\phi_{3}}=0.6$. In Case 2: $P_{\varphi_{1}}=0.4, P_{\varphi_{2}}=0.3$ and $P_{\varphi_{3}}=0.2$

average packet delay have been obtained for the relay network with spatially random relays. We show that, in the trust-aware network, the outage performance depends not only on diversity order but also error floor, which is very different from non trust aware network. The optimum target buffer lengths for best outage performance and minimum average delay have both obtained. The analysis shows that it is more necessary to control the buffer queuing lengths in the trust-aware network than in the non-trustaware network, because the trusts make the $S \rightarrow R_{k}$ and $R_{k} \rightarrow D$ transmissions more unbalanced.

\section{APPENDIX I}

If the target buffer lengths are set to $\Theta_{k}=0$ for all $k$, the buffer states can only be $\mathbf{0}$ or $\mathbf{0}^{(+1)}$ when the SNR goes to infinity. We can then re-group the buffer states and form a new Markov chain as is shown in Fig. 6.

The state transition matrix for Markov chain shown in Fig. 6 is given by

$$
\mathbf{A}=\left(\begin{array}{cc}
\beta & 1 \\
1-\beta & 0
\end{array}\right)
$$




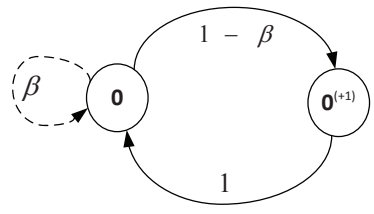

Fig. 6. Buffer state transition when the target buffer lengths are set to $\Theta_{k}=0$ for all $k$.

Thus we have

$$
\left(\begin{array}{c}
P_{\infty}(\mathbf{s}=\mathbf{0}) \\
P_{\infty}\left(\mathbf{s}=\mathbf{0}^{(+1)}\right)
\end{array}\right)=(\mathbf{A}-\mathbf{I}+\mathbf{B})^{-1} \mathbf{b}=\left(\begin{array}{c}
\frac{1}{2-\beta} \\
\frac{1-\beta}{2-\beta}
\end{array}\right)
$$

where $P_{\infty}(\mathbf{s}=\mathbf{0})$ and $P_{\infty}\left(\mathbf{s}=\mathbf{0}^{(+1)}\right)$ are the probabilities for buffer state at $\mathbf{0}$ and $\mathbf{0}^{(+1)}$ when the SNR goes to infinity respectively. Then we have

$$
\lim _{\bar{\lambda} \rightarrow \infty} \sum_{k=1}^{K} \bar{q}_{k}=1 \times P_{\infty}\left(\mathbf{s}=\mathbf{0}^{(+1)}\right)=\frac{1-\beta}{2-\beta}
$$

And from (26) the outage probability is given by

$$
\lim _{\bar{\lambda} \rightarrow \infty} P_{\text {out }}=\beta \cdot P_{\infty}(\mathbf{s}=\mathbf{0})=\frac{\beta}{2-\beta},
$$

Substituting (36) and (37) into (21) gives the average packet delay as

$$
\lim _{\bar{\lambda} \rightarrow \infty} \bar{D}=\lim _{\bar{\lambda} \rightarrow \infty}\left(1+\frac{2}{2-P_{\text {out }}} \sum_{k=1}^{K} \bar{q}_{k}\right)=2, \quad \Theta_{k}=0 \text { for all } k .
$$

\section{REFERENCES}

[1] J. Tang, G. Chen, and J. P. Coon, "Route selection based on connectivitydelay-trust in public safety networks," IEEE Systems Journal, 2018.

[2] X. Chen, B. Proulx, X. Gong, and J. Zhang, "Exploiting social ties for cooperative D2D communications:a mobile social networking case," IEEE/ACM Transactions on Networking, vol. 23, no. 5, pp. 1471-1484, Oct. 2015.

[3] G. Chen, J. Tang, and J. P. Coon, "Optimal routing for multi-hop socialbased $\mathrm{d} 2 \mathrm{~d}$ communications in the internet of things," IEEE Internet of Things Journal, 2018.

[4] J. M. Kleinberg, "Navigation in a small world," Nature, vol. 406, p. 845, 2000.

[5] D. Liben-Nowell, J. Novak, R. Kumar, P. Raghavan, A. Tomkins, and R. L. Graham, "Geographic routing in social networks," Proc. Nat. Acad. Sci. United States Amer, vol. 102, no. 33, pp. 11623-11628, 2005.

[6] H. Mao, W. Feng, Y. Zhao, and N. Ge, "Joint social-position relationship based cooperation among mobile terminals," IEEE Commun. Lett., vol. 18, no. 12, pp. 2165-2168, Dec. 2014.

[7] G. Chen, Z. Tian, Y. Gong, and J. A. Chambers, "Decode-and-forward buffer-aided relay selection in cognitive relay networks," IEEE Trans. Veh. Technology, vol. 63, no. 9, pp. 4723-4728, Mar. 2014.

[8] G. Chen, Z. Tian, Y. Gong, Z. Chen, and J. A. Chambers, "Max-ratio relay selection in secure buffer-aided cooperative wireless networks," IEEE Trans. Inform. Forensics and Security., vol. 9, no. 4, pp. 719-729, Apr. 2014.

[9] N. Zlatanov, R. Schober, and P. Popovski, "Buffer-aided relaying with adaptive link selection," IEEE J. Sel. Areas Commun., vol. 31, no. 8, pp. 1530-1542, Aug. 2012.

[10] N. Zlatanov and R. Schober, "Buffer-aided relaying with adaptive link selection - fixed and mixed rate transmission," IEEE Trans. Inform. Theory, vol. 59, no. 5, pp. 2816-2840, May 2013.

[11] V. Jamali, N. Zlatanov, and R. Schober, "Bidirectional buffer-aided relay networks with fixed rate transmission part I: Delay-unconstrained case," IEEE Transactions on Wireless Communications, vol. 14, no. 3, pp. 1323-1338, March 2015.

[12] V. Jamali, N. Zlatanov, and R. Schober, "Bidirectional buffer-aided relay networks with fixed rate transmission part II: Delay-constrained case," IEEE Transactions on Wireless Communications, vol. 14, no. 3, pp. 1339-1355, March 2015.

[13] T. Peng and R. C. de Lamare, "Adaptive buffer-aided distributed space-time coding for cooperative wireless networks," IEEE Trans. Commun., vol. 64, no. 5, pp. 1888-1900, May 2016.
[14] J. Gu, R. C. de Lamare, and M. Huemer, "Buffer-aided physical-layer network coding with optimal linear code designs for cooperative networks," IEEE Transactions on Communications, 2017.

[15] A. Ikhlef, D. S. Michalopoulos, and R. Schober, "Max-max relay selection for relays with buffers," IEEE Trans. Wireless Commun., vol. 11, no. 3, pp. 11241135, May 2012.

[16] I. Krikidis, T. Charalambous, and J. S. Thompson, "Buffer-aided relay selection for cooperative diversity systems without delay constraints," IEEE Trans. Wireless Commun., vol. 11, no. 5, pp. 1957-1967, May 2012.

[17] Z. Tian, Y. Gong, G. Chen, and J. A. Chambers, "Buffer-aided relay selection with reduced packet delay in cooperative networks," IEEE Trans. Veh. Tech., vol. 66, no. 3, pp. 2567-2575, Mar. 2017.

[18] C. Huang, J. Jiang, and S. Cui, "Asymptotic capacity of large fading relay networks with random node failures," IEEE Transactions on Communications, vol. 59, no. 8, pp. 2306-2315, 2011.

[19] H. Wang, S. Ma, and T. S. Ng, "On performance of cooperative communication systems with spatial random relays," IEEE Transactions on Communications, vol. 59, pp. 1190-1199, April 2011.

[20] J. R. Norris, "Markov chains," Cambridge University Press, 1998.

[21] A. Berman and R. J. Plemmons, "Nonnegative matrices in the mathematical sciences," Society of industrial and applied mathematics, 1994.

[22] J. D. C. Little, "A proof of the queueing formula: $L=\lambda \omega$," Operations Research, vol. 9, no. 3, pp. 383-388, 1961.

[23] Z. Tian, G. Chen, Y. Gong, Z. Chen, and J. A. Chambers, "Buffer-aided max-link relay selection in amplify-and-forward cooperative networks," IEEE Trans. Veh. Tech., vol. 64, no. 2, pp. 553-565, Feb. 2015.

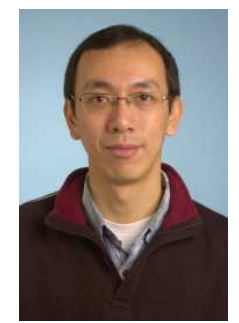

Yu Gong is with School of Electronic, Electrical and Systems Engineering, Loughborough University, UK, in July 2012. Dr Gong obtained his BEng and MEng in electronic engineering in 1992 and 1995 respectively, both at the University of Electronics and Science Technology of China. In 2002, he received his $\mathrm{PhD}$ in communications from the National University of Singapore. After $\mathrm{PhD}$ graduation, he took several research positions in Institute of Inforcomm Research in Singapore and Queens University of Belfast in the UK respectively. From 2006 and 2012, Dr Gong had been an academic member in the School of Systems Engineering, University of Reading, UK. His research interests are in the area of signal processing and communications including wireless communications, non-linear and non-stationary system identification and adaptive filters.

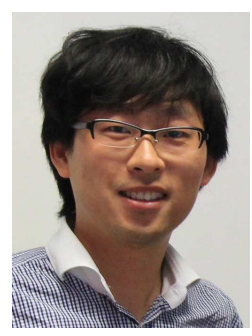

Gaojie Chen (S'09-M'12) received the B. Eng. and B. Ec. in Electrical Information Engineering and International Economics and Trade from the Northwest University, Shaanxi, China, in 2006, and the M.Sc (Distinction) and $\mathrm{Ph} . \mathrm{D}$ degrees from Loughborough University, Loughborough, UK, in 2008 and 2012, respectively, all in Electrical and Electronic Engineering. From 2008 to 2009 he worked, as a software engineering in DTmobile, Beijing, China, and from 2013 to 2018 as a Research Fellow at the Loughborough University, University of Surrey and University of Oxford, U.K. He is currently a Lecturer with the Department of Engineering, University of Leicester, U.K. His current research interests include wireless communications, cooperative communications, secrecy communication and random geometric networks.

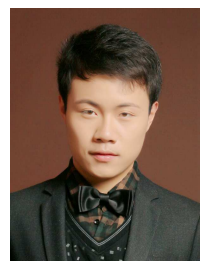

Tian Xie received the BEng in electronic and information engineering at Northwestern Polytechnical University, Shaanxi, China, in 2016 and the M.sc from Loughborough University, UK, in 2017. He currently works for Ericsson Shanghai as a software developer in the project of session border gateway. 